\title{
From Micro to Nano: Correlative 3D Microscopies for Analysis of Biointerfaces.
}

\author{
Anders Palmquist ${ }^{1}$ and Kathryn Grandfield ${ }^{2}$ \\ ${ }^{1 .}$ Department of Biomaterials, Sahlgrenska Academy, University of Gothenburg, Sweden. \\ 2. Department of Materials Science and Engineering, McMaster University, Hamilton, Canada.
}

Orthopaedic and dental implant materials rely heavily on their ability to integrate with bone tissue for successful clinical outcomes, where osseointegration refers to the direct bonding between titanium implants and bone [1]. Evaluating osseointegration, particularly at early time points, presents a variety of challenges. In order to correlate the bone growth mechanisms at implant surfaces to macroscale implant biocompatibility various three-dimensional (3D) microscopies have been utilized, including XRay microcomputed tomography (micro-CT), focused ion beam microscopy (FIB) and Z-contrast electron tomography.

In this work, screw-shaped titanium implants, $2.0 \mathrm{~mm}$ in diameter and $2.3 \mathrm{~mm}$ in length, were manufactured and treated with a biomimetic hydroxyapatite surface coating according to [2]. Implants were placed in the tibia of rats and removed with surrounding bone after 7 and 28 days, fixed in glutaraldehyde and embedded into blocks for further analyses.

Full $360^{\circ}$ rotation X-ray micro-CT scans were performed (Skyscan 1172, Bruker Corporation) with a source voltage and current of $85 \mathrm{kV}$ and $114 \mu \mathrm{A}$, respectively, and a $3.96 \mu \mathrm{m}$ pixel size. Bone apposition on the implant surface in as little as 7 days was observed, Figure 1, dominated by less organized and mineralized woven bone tissue and some remaining bone fragments from the surgery. As expected, increased organization and maturation of the bone tissue with increasing implantation time was also noted.

FIB played a critical role in investigating the material during both in vitro testing stages and preparation of specimens for TEM after in vivo studies. The site-specific technique, which can also be used for 2D and $3 \mathrm{D}$ visualization of bone interfaces [3], was used to prepare intact specimens for TEM and electron tomography.

Electron tomograms of the titanium substrate-coating interface (Figure 2) and the bone-coating interface were collected (Titan 80-300, FEI Company) using a single-axis linear tilt scheme at 300kV with the Advanced Tomography Holder (Model 2020, Fischione Instruments). Automated focusing, image shifting, and acquisition of high-angle annular dark-field (HAADF) STEM images over an angular range of $\pm 65^{\circ}$ was achieved using the FEI Explore $3 \mathrm{D}$ software. The $3 \mathrm{D}$ reconstructions were computed using a simultaneous iterative reconstruction technique, with 20 iterations, in FEI's Inspect3D. Models for 3D visualization were created in (Amira Resolve RT FEI, Visage Imaging Inc.). Resolving the implant-bone interfaces with the higher resolution afforded by electron tomography versus X-ray based tomography, allowed us to investigate the role of the nanostructured surface coating and visualize the organization of collagen and hydroxyapatite crystals at the immediate implant interface.

Utilizing three-dimensional techniques ranging from X-ray micro-CT to FIB to Z-contrast electron tomography has allowed us to correlate the macroscale bone-implant contact to the nanometer scale 
ultrastructure of bone constituents at the implant interface. Combining three-dimensional information at various length scales provides insight into the mechanisms of early bone formation at an implant interface and overall implant integration [4].

\section{References:}

[1] P-I Brånemark et al, Scand J Plast Reconstr Surg 3 (1969), p. 81.

[2] A Thorfve et al, Acta Biomaterialia 10 (2014), p. 1451.

[3] LA Giannuzzi at al, J Oral Maxillofac Surg 65 (2007), p. 737.

[4] The electron microscopy work described in this paper was performed at the Canadian Centre for Electron Microscopy at McMaster University; a facility supported by NSERC and other government agencies.

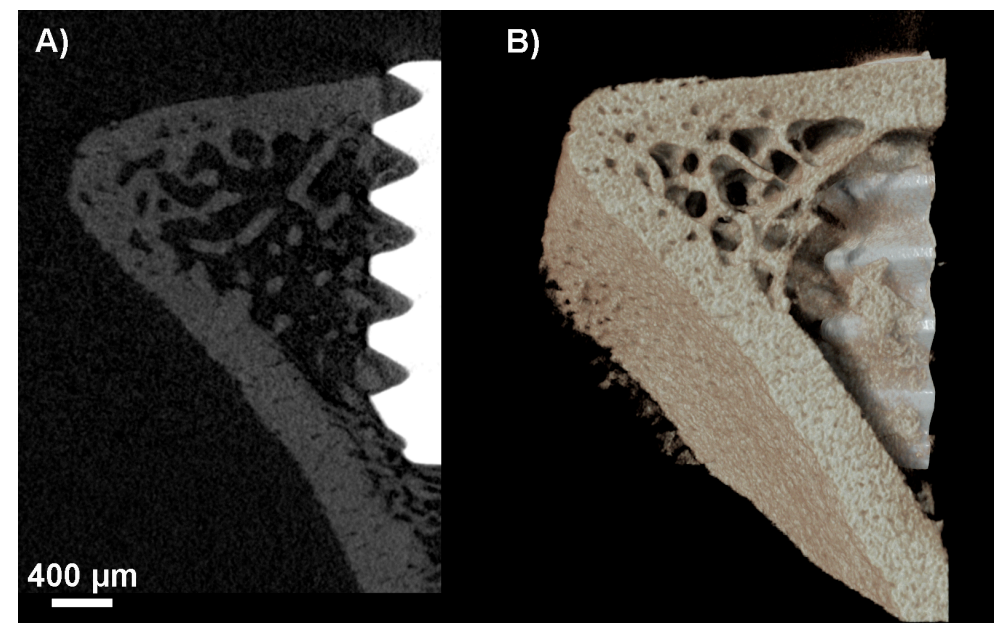

Figure 1. Micro-CT scans of the microscale implant-bone interface after 7 days in vivo, showing bone trabeculae and early deposition of bone on the implant surface in (a) a reconstructed virtual section and (b) complete three-dimensional rendering.

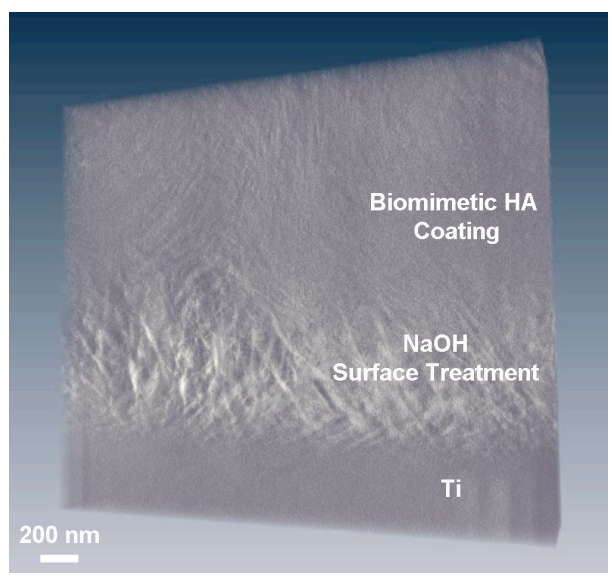

Figure 2. Z-contrast electron tomography of the titanium implant shows fine details of the surface treatment structure (and bone-implant interface, not shown), with a superior resolution than micro-CT. 